\title{
Maximizing Potentially Avoidable Hospitalizations and Cost Savings Beyond Targeting the Most Costly Patients
}

Mariana Simons ${ }^{1}$, PhD; Sara Golas ${ }^{2}$, PhD; Stephen Agboola ${ }^{2}$, MPH, MD; Jorn op den Buijs ${ }^{1}$, PhD; Jennifer Felsted ${ }^{2}$, PhD; Nils Fischer ${ }^{2}$, MPH; Allison Orenstein ${ }^{3}, \mathrm{MPH}$

${ }^{1}$ Philips Research, Eindhoven, Netherlands

${ }^{2}$ Connected Health Innovation, Partners Healthcare, Boston, MA, United States

${ }^{3}$ Philips Lifeline, Framingham, MA, United States

\section{Corresponding Author:}

Mariana Simons, PhD

Philips Research

34 High Tech Campus

Eindhoven,

Netherlands

Email: mariana.simons@philips.com

\section{Abstract}

Background: Many health care organizations use value-based care strategies that include population health management programs and data analytics to stratify their population and identify high-risk and high-cost patients. Most of these programs target the top 5\% most expensive patients. However, little is known about these patients prior to reaching the top 5\% of cost, or how their characteristics change over time. To address these gaps, we analyzed the differences in characteristics of patients from 3 different cost segments over 5 years (2011-2015).

Objective: To evaluate potentially avoidable hospitalizations and associated savings in the health care cost of older patients using Personal Emergency Response Service (PERS).

Methods: We conducted a retrospective, longitudinal, multicenter study to evaluate potentially avoidable hospitalizations of 2643 older patients over 5 years (2011-2015). All patients had at least one inpatient and/or outpatient encounter, and at least one episode of home health care during the study period. Additionally, all patients used PERS at home anytime during the study period. We ranked patients by their annual health care cost and then grouped them into the following segments for each respective year: T-segment constitutes the top 5\% most expensive patients; M-segment comprises the middle 45\% of patients; B-segment includes the bottom 50\% least expensive patients. We then evaluated differences in the characteristics of patients in the B-, Mand T-cost segments in each study year. Continuous variables were compared by $t$ test (two-tailed) for normally distributed variables and Kruskal-Wallis Rank Sum test for skewed variables. The chi-square test was used for categorical variables.

Results: The three cost segments differed significantly each year $(P<.05)$ with respect to: demographics (age, education), PERS utilization (Incidents, ER transport), health care utilization (hospitalizations, length of stay, 30-, 90-, and 180-day readmissions, outpatient encounters) and medical conditions (number of conditions, Charlson Comorbidity Index). Further, we analyzed the number of potentially avoidable hospitalizations (as defined by CMS) and associated cost savings in each segment. All hospitalizations occurred among patients in the T- and M-segments while the B-segment was hospitalization-free each year. The percentage of avoidable hospitalizations in the M-segment compared with the T-segment was 3 times greater (75\% vs $25 \%$, $P<.001)$. While the potential cost saving from avoidable hospitalizations in the entire population increased from $\$ 3.0 \mathrm{M}$ to $\$ 8.2 \mathrm{M}$ (2011-2015), the majority of these cost savings were in the M-segment compared with the T-segment $(60 \%$ vs $40 \%, P<.001)$.

Conclusions: Although many health care organizations target intensive and costly interventions to their most expensive patients, this analysis suggests there is untapped potential to control costs and improve care beyond focusing on the highest cost patients. Namely, targeting patients in the middle cost segment may offer great opportunity for population management programs to maximize both potentially avoidable hospitalizations and cost savings.

(iproc 2018;4(2):e11731) doi: $10.2196 / 11731$

\section{KEYWORDS}

patient segmentation; avoidable hospitalizations; healthcare cost analysis 
Edited by T Hale; this is a non-peer-reviewed article. Submitted 30.07.18; accepted 29.08.18; published 17.09.18.

Please cite as:

Simons M, Golas S, Agboola S, op den Buijs J, Felsted J, Fischer N, Orenstein A

Maximizing Potentially Avoidable Hospitalizations and Cost Savings Beyond Targeting the Most Costly Patients iproc 2018;4(2):e11731

URL: http://www.iproc.org/2018/2/e11731/

doi: $\underline{10.2196 / 11731}$

PMID:

CMariana Simons, Sara Golas, Stephen Agboola, Jorn op den Buijs, Jennifer Felsted, Nils Fischer, Allison Orenstein. Originally published in Iproceedings (http://www.iproc.org), 17.09.2018. This is an open-access article distributed under the terms of the Creative Commons Attribution License (https://creativecommons.org/licenses/by/4.0/), which permits unrestricted use, distribution, and reproduction in any medium, provided the original work, first published in Iproceedings, is properly cited. The complete bibliographic information, a link to the original publication on http://www.iproc.org/, as well as this copyright and license information must be included. 